\title{
Interfacial Microstructure and Hardness of Magnetic Pulse Welded Copper/Nickel Lap Joint
}

\author{
Mitsuhiro Watanabe ${ }^{1, * 1}$, Kentaro Ishiuchi ${ }^{2, * 2}$ and Shinji Kumai ${ }^{3}$ \\ ${ }^{1}$ Department of Precision Machinery Engineering, College of Science and Technology, Nihon University, Funabashi 274-8501, Japan \\ ${ }^{2}$ Tokyo Institute of Technology, Yokohama 226-8502, Japan \\ ${ }^{3}$ Department of Materials Science and Engineering, School of Materials and Chemical Technology, Tokyo Institute of Technology, \\ Tokyo 152-8552, Japan
}

Copper/nickel joint was fabricated using magnetic pulse welding and the interfacial microstructure was examined. Hardness change close to the welding interface was investigated by using a nanoindentation tester. Characteristic wavy morphology was formed at the welding interface. TEM observations and STEM-EDX analyses revealed that approximately $100 \mathrm{~nm}$-diameter-fine grains of Cu-Ni solid solution were formed at both of copper and nickel regions in the vicinity of the welding interface. Remarkable hardness increase was detected at the vicinity of the welding interface in the nickel side, whereas hardness was constant in the copper side. The hardness increase is considered to be due to the combined effect of solid-solution strengthening and grain refinement strengthening. [doi:10.2320/matertrans.M2017289]

(Received September 25, 2017; Accepted December 18, 2017; Published February 2, 2018)

Keywords: magnetic pulse welding, copper/nickel joint, interface, microstructure, hardness

\section{Introduction}

Solid-state welding is effective method for dissimilar metal joining. This is because heat input for the welding is lower than that of fusion welding and mechanical mixing of the welded metals produces characteristic microstructure at the welding interface. Magnetic pulse welding is one of the solid-state welding method and can be achieved within 10 microseconds with a negligible temperature increase ${ }^{1,2)}$.

Schematic diagram of the principle of magnetic pulse welding is shown in Fig. 1. The two plates are placed above the coil, with a small gap between them. The plate set close to the coil is called the "flyer plate" and the plate above it, which is fixed firmly in place, is referred to as the "parent plate". When a discharge pulse passes through the coil, it induces a high-density magnetic flux around the coil. The generated magnetic flux lines intersect with the flyer plate, and in accordance with Lentz's law, eddy currents are excited in

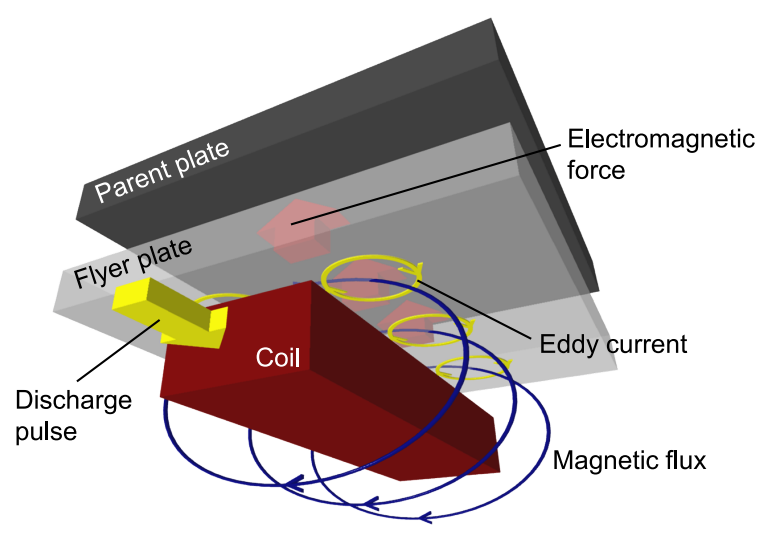

Fig. 1 Schematic diagram of magnetic pulse welding.

\footnotetext{
${ }^{* 1}$ Corresponding author, E-mail: watanabe.mitsuhiro@nihon-u.ac.jp

${ }^{* 2}$ Graduate Student, Tokyo Institute of Technology. Present address:

Nissan Motor Co., Ltd., Atsugi 243-0192, Japan
}

the surface of the flyer plate adjacent to the coil. In accordance to Fleming's left-hand rule, the eddy current and the magnetic flux generated around the coil induce an electromagnetic force upward. The flyer plate bulges toward the parent plate at a high velocity by the electromagnetic force (Fig. 2(a)). Collision angle increases continuously when the collision point moves to outside (Fig. 2(b) and 2(c)). It is known that welding of metals is achieved when the collision angle and collision velocity are suitable for the metals ${ }^{3)}$. Therefore, in magnetic pulse welded joint, the welding is achieved two parts where the collision angle and collision velocity are suitable ${ }^{4)}$.

Dissimilar metal combinations such as aluminum/ steel ${ }^{5-8)}$, aluminum/copper ${ }^{9,10)}$, and aluminum/magnesium ${ }^{11,12)}$ has been welded using magnetic pulse welding and high strength dissimilar metal joints were obtained. These welding interfaces exhibit characteristic wavy morphology and intermediate layer is formed along the wavy interface in some metal combinations. The intermediate layer is composed of mixture of fine grains and amorphous phase.

The present authors reported collision behavior of two and multiple metal plates during magnetic pulse welding process $^{4,13)}$. We revealed that the wavy interface is formed by high-speed oblique collision and the wavy interfacial morphology depends on collision velocity and collision angle. Metal jet emission from the collision point was also directly observed by using the high-speed video imaging. Kakizaki reported that composition of the metal jet emission behavior is controlled by the relative density difference between two metals to be welded ${ }^{14)}$. The metal jet is emitted from both metal surfaces when the density difference is small or zero. While, the metal jet emission from the metal with lower density occurs when the density difference is large. Ishii observed explosive welding process of several dissimilar metal combinations by using a streak camera and discussed the emission direction of metal jet ${ }^{15}$. In this paper, the metal plates are treated as fluids and they suggested that the metal jet is emitted from the metal surface with a high 
density.

Metal combinations that have large density difference and form intermetallic compounds have been the target of investigation in the previous studies. In order to understand the formation mechanism of the interfacial microstructure produced by magnetic pulse welding, it is necessary to investigate the welding interface for other kinds of metal combinations. In this study, copper/nickel joint was selected and the interfacial microstructure was examined. Copper and nickel are miscible in all the composition range ${ }^{16)}$ and the density difference is extremely small $\left(\rho_{\mathrm{Cu}}=8.93 \mathrm{Mg} / \mathrm{m}^{3}, \rho_{\mathrm{Ni}}=\right.$ $\left.8.90 \mathrm{Mg} / \mathrm{m}^{3}\right)$. Therefore, the combination of copper and nickel is contrastive to the aluminum/iron and aluminum/ copper dissimilar-metal combinations. Also, temperature increase generated during the welding process is known to induce microstructural change of the welding interface and to affect the mechanical properties of the joint. Therefore, to know the mechanical properties of microstructure in the vicinity of the welding interface is important. In this study, hardness evaluation was performed. In the magnetic pulse welded joint, the width of microstructural change from the
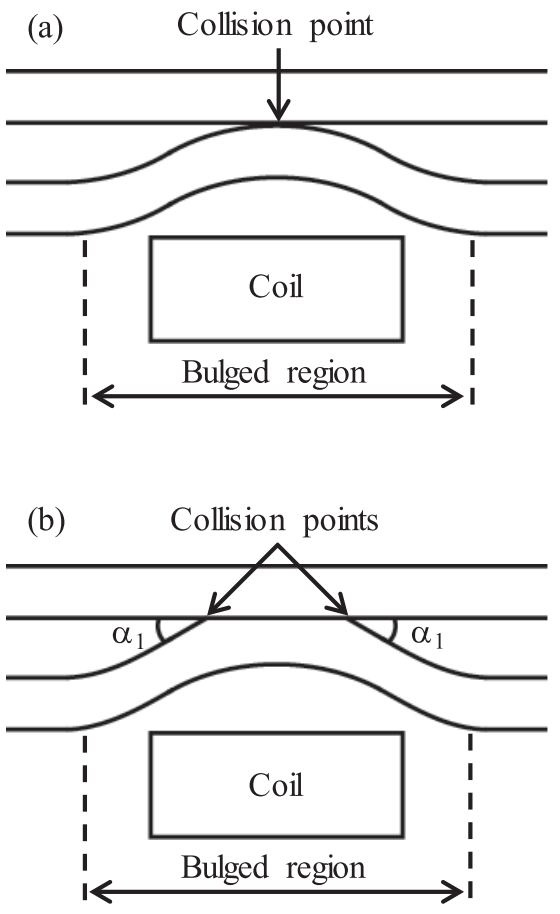

(c) Collision points

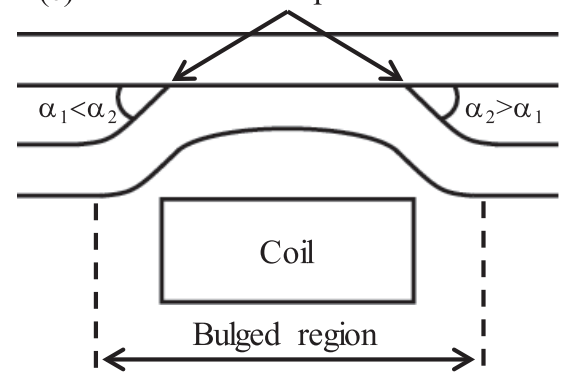

Fig. 2 Schematic diagrams of collision process during magnetic pulse welding ${ }^{4}$. (a) Initial collision. (b), (c) Collision with angle. welding interface has been reported to be usually several micrometers ${ }^{6,17)}$. Nanoindentation test is suitable for hardness measurement of small region of microstructure. Therefore, hardness in the vicinity of the welding interface was precisely investigated using the nanoindentation test and hardness mechanism was discussed.

\section{Experimental Procedure}

A pure copper (oxygen free copper, 99.99 mass \%, hereafter $\mathrm{Cu})$ plate $(100 \mathrm{~mm} \times 100 \mathrm{~mm} \times 1.2 \mathrm{~mm})$ and a pure nickel $(99.74$ mass $\% \mathrm{Ni}$, hereafter $\mathrm{Ni})$ plate $(100 \mathrm{~mm} \times$ $100 \mathrm{~mm} \times 1.0 \mathrm{~mm}$ ) were used in this study. Figure 3 shows optical micrographs of the grain structure of (a) $\mathrm{Cu}$ and (b) $\mathrm{Ni}$ plate. The original shape of the grain was fibrous morphology. Before welding, the plate surfaces were rinsed in acetone with an ultrasonic cleaner and were dried in air.

For generation of electromagnetic force used for highspeed collision, a discharge circuit including a capacitor (400 $\mu \mathrm{F}$ ) for charging of electrical energy, a discharge gap switch, and a coil was used ${ }^{18)}$. A Cu plate was set over the coil as a flyer plate and a $\mathrm{Ni}$ plate was placed above the $\mathrm{Cu}$ plate with a $0.8 \mathrm{~mm}$ gap as a parent plate. $\mathrm{Cu} / \mathrm{Ni}$ welding was performed by the electromagnetic force generated at the charging energy of $3.3 \mathrm{~kJ}$.

The welding interface was observed by using an optical microscope and a transmission electron microscope (TEM, H-1250 (acceleration voltage: $1000 \mathrm{kV})$ ). The chemical composition was analyzed by using electron probe microanalysis (EPMA, JXA-8900 (acceleration voltage: $20 \mathrm{kV}$ )) and energy dispersive X-ray spectrometry equipped with a scanning transmission electron microscope (STEM-EDX, EM-002BF (acceleration voltage: $200 \mathrm{kV}$ )). The EPMA

(a)

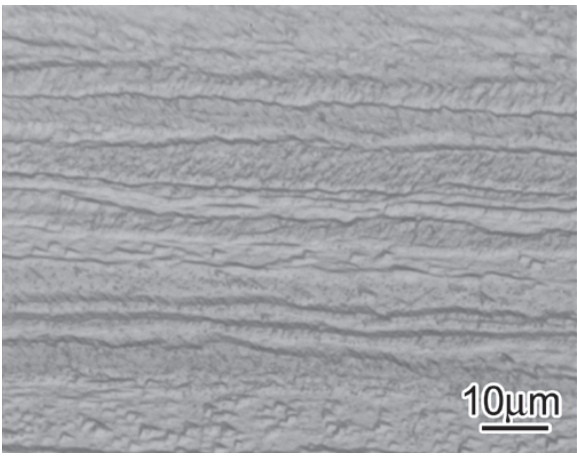

(b)

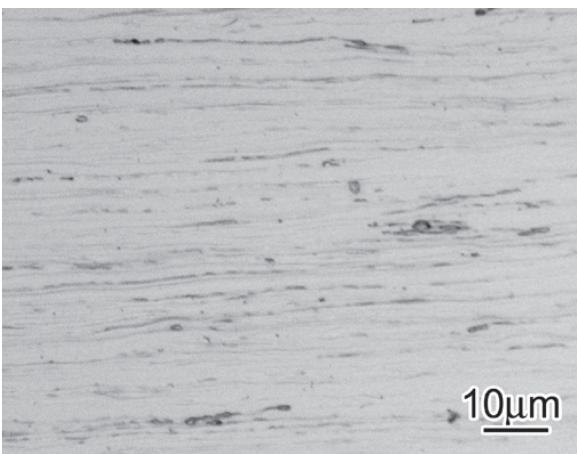

Fig. 3 Optical micrographs of grain structure of original metal plates. (a) Cu plate. (b) Ni plate. 
analysis was conducted in continuous point analysis mode with analysis interval of $0.5 \mu \mathrm{m}$ using analysis diameter of approximately $1 \mu \mathrm{m}$. The TEM and STEM samples were prepared by focused ion beam (FIB, FB-2100 (acceleration voltage: $40 \mathrm{kV}$ )) method using $\mathrm{Ga}$ ions. Surface of the thinned samples by the FIB method was finally polished by a low-energy ion milling using Ar ion (acceleration voltage: $250 \mathrm{~V}$ ) for removing the damage layer caused during the FIB process.

The local hardness change after the magnetic pulse welding was evaluated by a nanoindentation tester (ENT-1100A). The tip of the indenter was Berkovich-shape and the applied load was $100 \mathrm{mgf}$. The indentation measurements were performed in the area $(15 \mu \mathrm{m} \times 7 \mu \mathrm{m})$ including the welding interface. The measurement intervals were $5 \mu \mathrm{m}$ to the perpendicular direction of the welding interface and $3 \mu \mathrm{m}$ to the parallel direction.

\section{Results and Discussion}

\subsection{Microstructure of $\mathrm{Cu} / \mathrm{Ni}$ welding interface}

Figure 4 shows an optical micrograph of cross-section of the welding interface. The upper dark-contrast side is $\mathrm{Ni}$ and the lower bright-contrast side is $\mathrm{Cu}$. The welding interface exhibited characteristic wavy morphology. Drastic contrast change was observed at the $\mathrm{Cu} / \mathrm{Ni}$ interface, and no intermediate layer with different contrast was observed.

Wavy welding interface which is similar to the magnetic pulse welded interface has been observed at explosively welded interface ${ }^{19}$ ). Since the explosively welding is achieved by high-speed oblique collision like the magnetic pulse welding, formation mechanism of the wavy interface is considered to common. Several formation models of the wavy interface in explosively welded joints have been proposed. Bahrani ${ }^{20)}$ and Cowan ${ }^{21)}$ suggested that oscillation behavior of the metal jet emitted from collision point results in formation of continuous asperity at plate surface. Reid ${ }^{22)}$ paid attention the vortex formed at crest of the interfacial wave and proposed that wavy interface is produced by formation of von Kármán vortex street. There has been no consensus on formation model of the interfacial wave yet.

Figure 5 shows composition change across the welding interface detected by using EPMA analysis. The left-hand side is $\mathrm{Cu}$ and the right-hand side is Ni. The zero on the hor-

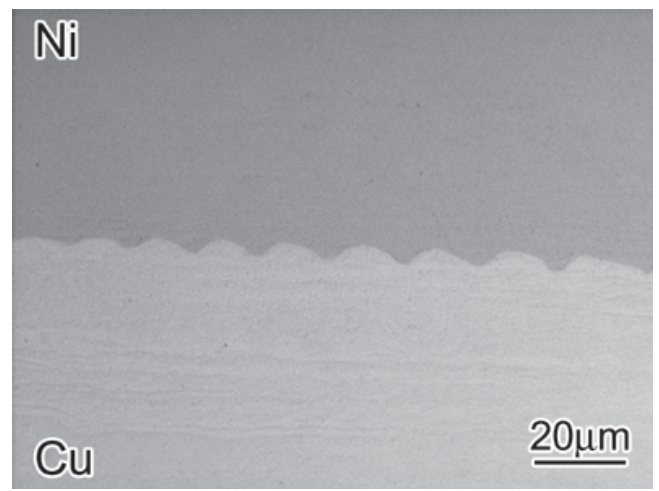

Fig. 4 Optical micrograph of cross-sectional $\mathrm{Cu} / \mathrm{Ni}$ welding interface. izontal axis is the welding interface. Composition ratio of $\mathrm{Cu}$ and $\mathrm{Ni}$ elements drastically changed at the welding interface, while, the composition was constant in the area over $1 \mu \mathrm{m}$ from the welding interface. Since the beam diameter of the EPMA analysis is more than $1 \mu \mathrm{m}$, composition change in the area within $1 \mu \mathrm{m}$ from the welding interface is not detectable correctly. Further precise composition measurements are needed for understanding the local composition change at the welding interface, however, the present result shows that at least no compositional change occurred in both $\mathrm{Cu}$ and $\mathrm{Ni}$ in the range over a few micrometers away from the welding interface.

TEM observation was performed for precise investigation of the welding interface. Figure 6(a) shows a TEM-brightfield image of the welding interface. The upper side is $\mathrm{Ni}$ and the lower side is $\mathrm{Cu}$. A sound interface with no voids and inclusions was formed. Note that a crack in the figure was a damage caused by the FIB process. In order to locate the interface position between $\mathrm{Cu}$ and $\mathrm{Ni}$, STEM-EDX analysis was performed. The X-ray intensity maps of $\mathrm{Cu}$ and $\mathrm{Ni}$ are shown in Figs. 6(b) and 6(c), respectively. Strong $\mathrm{Cu}$ and $\mathrm{Ni}$ signals were detected over the entire $\mathrm{Cu}$ and $\mathrm{Ni}$ regions, respectively. According to this result, we could know that the welding interface of $\mathrm{Cu} / \mathrm{Ni}$ is located as shown by a white broken curve in Fig. 6(a).

The area with dark-contrast which is different from bright area showing original fibrous grain structure was clearly observed in Ni-side (indicated by region A in Fig. 6(a)) and $\mathrm{Cu}$-side (indicated by region $\mathrm{B}$ in Fig. 6(a)). STEM-EDX maps indicate that both $\mathrm{Cu}$ and $\mathrm{Ni}$ elements coexist in the region $\mathrm{A}$ and the signal intensity of $\mathrm{Ni}$ is stronger than that of $\mathrm{Cu}$. The $\mathrm{Cu}$ signal intensity decreased gradually with approaching toward the region $\mathrm{A}$ from $\mathrm{Cu}$. The $\mathrm{Ni}$ signal shows the opposite trend. The Ni intensity was detected at $\mathrm{Cu}$ region over $1 \mu \mathrm{m}$ from the $\mathrm{Cu} / \mathrm{Ni}$ interface.

An enlarged TEM-bright-field image of the region $\mathrm{A}$ is shown in Fig. 7(a). Extremely fine crystal grain structure was formed at this region. The size of the grain was around 100-200 nm. The diffraction pattern taken from this region is shown in Fig. 7(b). It consists of several rings of sharp spots. This indicates the existence of fine crystal grains with random orientations. The average lattice parameter $(0.3541 \mathrm{~nm})$ obtained from these ring patterns was little larger than that of pure $\mathrm{Ni}\left(0.3524 \mathrm{~nm}^{23)}\right)$ and was smaller

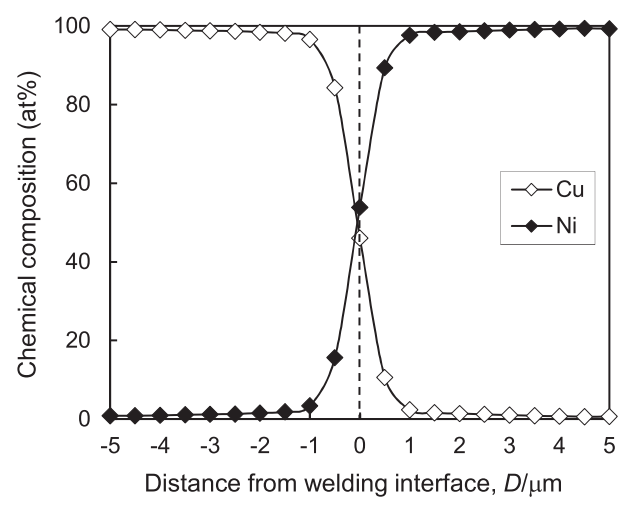

Fig. 5 Change in chemical composition across the welding interface measured by using EPMA. 
(a)

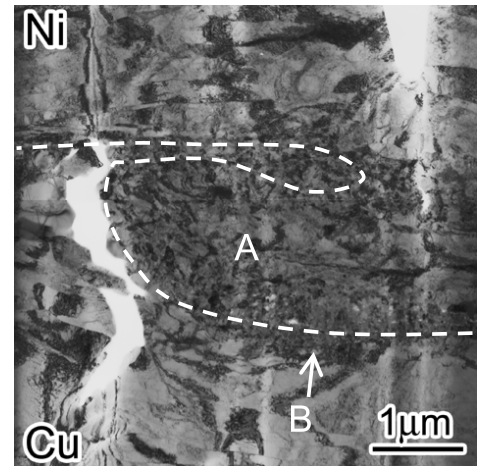

(b)

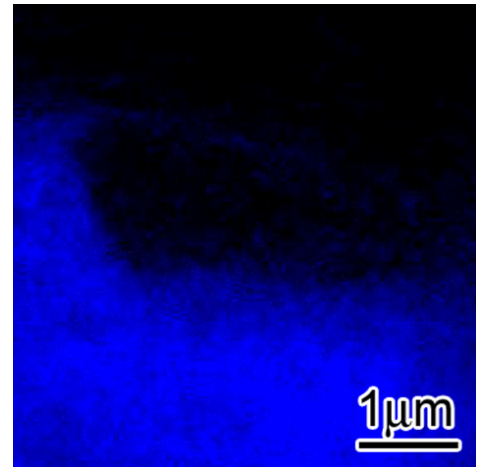

(c)

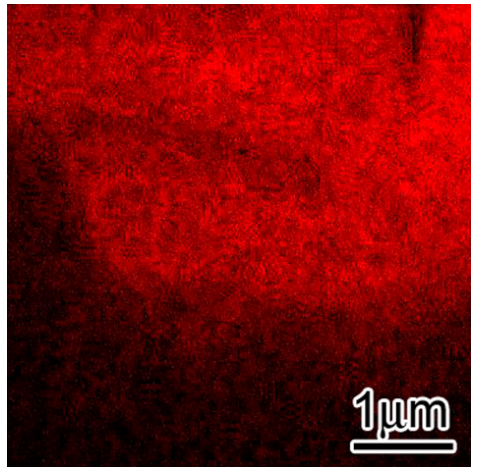

Fig. 6 (a) TEM-bright-field image of welding interface and STEM-EDX maps of (b) $\mathrm{Cu}$ and (c) Ni.

(a)

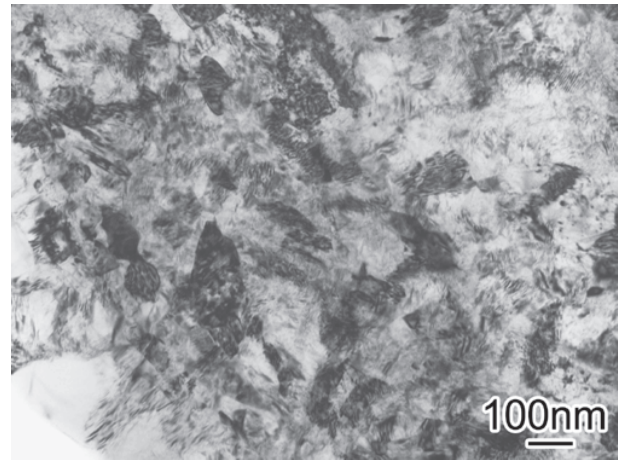

(b)

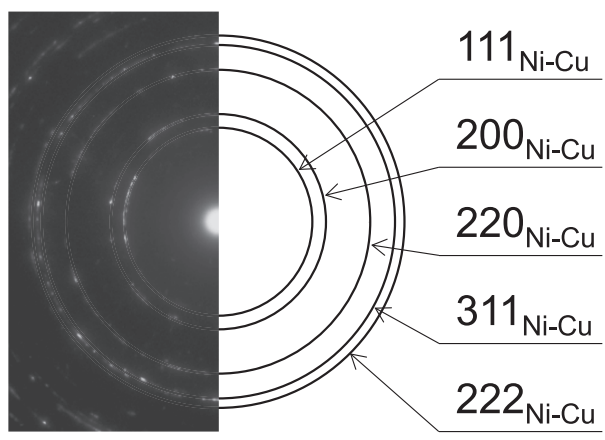

Fig. 7 (a) TEM-bright-field image of region A indicated in Fig. 6(a). (b) Electron diffraction pattern taken from region $\mathrm{A}$.

than that of pure $\mathrm{Cu}\left(0.3615 \mathrm{~nm}^{24)}\right)$. Since the lattice parameter of the $\mathrm{Cu}-\mathrm{Ni}$ alloy is proportional to concentration of the solute element ${ }^{25}$, $\mathrm{Cu}$ concentration of the alloy formed in region A was estimated to be $17 \%$ from the lattice parameter. This suggests that fine $\mathrm{Ni}-17 \% \mathrm{Cu}$ solid-solution grains were formed at region within approximately $2 \mu \mathrm{m}$ from the welding interface in Ni-side. In contrast to that, the fibrous grain structure comparable to the original structure (Fig. 3(b)) was observed over $2 \mu \mathrm{m}$ from the welding interface in Ni-side.

Figure 8(a) shows a TEM-dark-field image of the region B in Fig. 6(a), and Fig. 8(b) shows selected diffraction pattern taken from this region. As well as the Ni-side, extremely fine crystal grains with approximately $100 \mathrm{~nm}$ diameter were formed along the welding interface. The lattice parameter obtained by the ring diffraction patterns $(0.3592 \mathrm{~nm})$ was smaller than that of pure $\mathrm{Cu}$. The estimated composition of the solid solution was $\mathrm{Cu}-27 \% \mathrm{Ni}$. The fine grain structure was formed within approximately $500 \mathrm{~nm}$ from the welding interface in $\mathrm{Cu}$-side. The width of the fine grain structure area in $\mathrm{Cu}$-side was narrower than that of $\mathrm{Ni}^{-}$ side. No structural change was observed over $500 \mathrm{~nm}$ away from the welding interface in $\mathrm{Cu}$ region.

The extremely fine $\mathrm{Cu}-\mathrm{Ni}$ solid-solution grains were formed at the welding interface. Such the fine grain is considered to be formed either by rapid solidification from liquid state or heavily plastic deformation of solid state ${ }^{26,27)}$ and it depends on whether the interface was melted or not. Therefore, temperature increase at the welding interface is a key to know the formation mechanism of microstructure in this region. Direct temperature measurement during the welding process is difficult because magnetic pulse welding is completed in extremely short time (normally within $\left.10 \mu \mathrm{s}^{18)}\right)$. In addition, quite local temperature increase at the welding interface is expected in the magnetic pulse welding. Numerical simulation is a good tool to understand the temperature during the welding process. Nishiwaki investigated temperature at the wavy aluminum/copper welding interface during magnetic pulse welding using smoothed particle hy- 
(a)

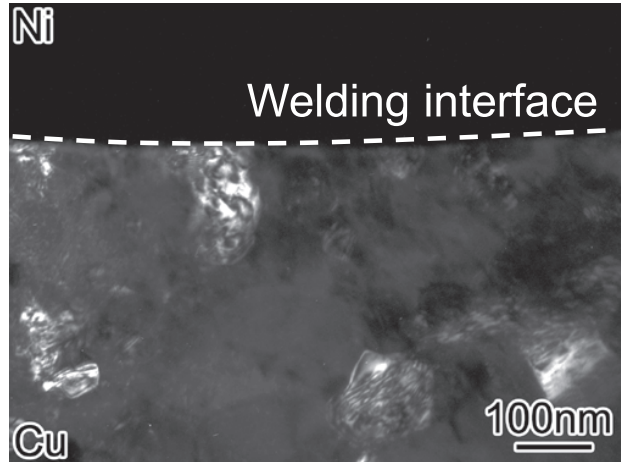

(b)

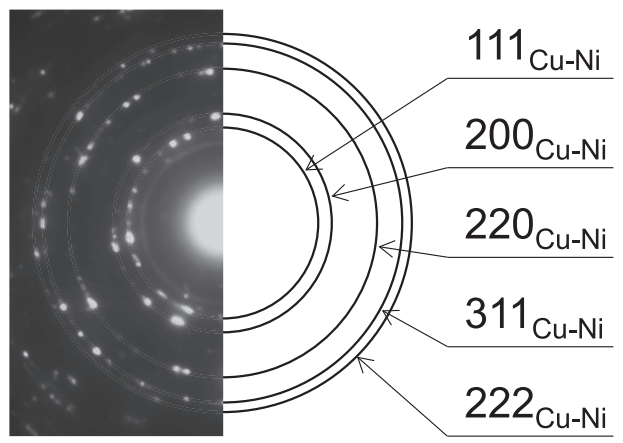

Fig. 8 (a) TEM-dark-field image of region B indicated in Fig. 6(a). White broken curve indicates the welding interface. (b) Selected area diffraction pattern taken from region $\mathrm{B}$.

drodynamics ${ }^{28)}$. They reported that the temperature of vortex zone produced at the wavy interface exceeds the melting points of copper $(2899 \mathrm{~K})$. Because the position of the vortex zone produced at aluminum/copper interface correspond to that of the fine $\mathrm{Cu}-\mathrm{Ni}$ solid-solution grain region observed in this study and the melting points of $\mathrm{Cu}(1356 \mathrm{~K})$ and $\mathrm{Ni}$ $(1726 \mathrm{~K})$ is lower than $2899 \mathrm{~K}$, the region of the fine $\mathrm{Cu}-\mathrm{Ni}$ solid-solution grains is assumed to be melt. Also, Sapanathan showed temperature history at magnetic pulse welded interface ${ }^{29)}$ and according to its temperature history, the cooling rate of the welding interface can be estimated to be $10^{7}-10^{8} \mathrm{~K} / \mathrm{s}$. Therefore, the fine $\mathrm{Cu}-\mathrm{Ni}$ solid-solution grain region is assumed to be formed by melting once and then rapidly solidifying.

\subsection{Hardness distribution at the welding interface}

To investigate local hardness change caused by magnetic pulse welding, nanoindentation test was performed at the welding interface. Figure 9(a) shows a SEM-backscattered electron image of the area provided for hardness measurement. A series of small indentations were clearly observed at the welding interface. Relationship between the hardness and distance from the welding interface is shown in Fig. 9(b). The left-hand side is $\mathrm{Cu}$ and the right-hand side is $\mathrm{Ni}$. The zero on the horizontal axis indicates the welding interface. Closed diamond and circle symbols located at both vertical axes of the figure are hardness values of the original $\mathrm{Cu}$ and $\mathrm{Ni}$, and they are $2.10 \mathrm{GPa}$ and $3.13 \mathrm{GPa}$, respectively. The hardness of $\mathrm{Cu}$ was constant and was comparable to the hardness of the original $\mathrm{Cu}$ matrix. In the $\mathrm{Ni}$-side, hardness was almost constant and was equal to that of the original Ni matrix at the area over $2 \mu \mathrm{m}$ from the welding (a)

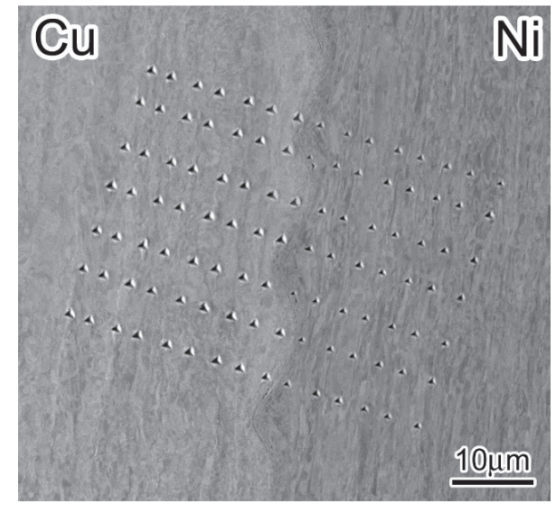

(b)

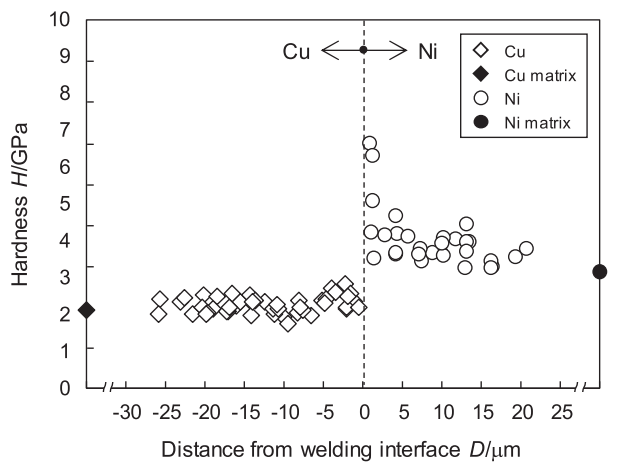

Fig. 9 Hardness distribution at welding interface. (a) Measurement region. (b) Relationship between hardness and distance from welding interface.

interface. However, a remarkable hardness increase was observed in the area within $2 \mu \mathrm{m}$ from the interface.

Remarkable hardness increase was detected only in the $\mathrm{Ni}$-side close to the welding interface. Microstructural observation revealed the formation of extremely fine $\mathrm{Ni}$ $17 \% \mathrm{Cu}$ solid-solution grains (Fig. 7). Since the width of the fine grain structure corresponds to that of the hardness increase region, both solid-solution strengthening and grain refinement strengthening are possible reasons of the increased hardness. Effect of work hardening should be taken into account because the magnetic pulse welded interface was heavily deformed by the applied impact pressure.

Effect of solute element on hardening of $\mathrm{Cu}-\mathrm{Ni}$ alloy has been investigated ${ }^{30)}$. The hardness of $\mathrm{Cu}-\mathrm{Ni}$ alloy gradually increases with increasing the amount of $\mathrm{Ni}$ from that of pure $\mathrm{Cu}(34 \mathrm{HB})$, but it shows the maximum value $(81 \mathrm{HB})$ at $\mathrm{Ni}$ amount of $58 \%$ and then gradually decreased to that of pure $\mathrm{Ni}(61 \mathrm{HB})$. The hardness of $\mathrm{Ni}-17 \% \mathrm{Cu}$ alloy is approximately $75 \mathrm{HB}$ and this value is found to be $123 \%$ higher than that of pure Ni. Since Berkovich hardness of an original pure Ni matrix used in this study is $3.13 \mathrm{GPa}$, the Berkovich hardness value by solid-solution strengthening effect should be $3.85 \mathrm{GPa}$ which is estimated by multiplying $3.13 \mathrm{GPa}$ by 1.23. However, in this study, hardness over $6.00 \mathrm{GPa}$ was obtained in $\mathrm{Ni}$-side close to the welding interface. Therefore, the other strengthening mechanism is considered to work. Grain refinement is known to show strengthening effect according to Hall-Petch relation. According to the relation, stress of the material is proportional to the reciprocal of the grain size. Since hardness is known to have good relation to stress, relationship between hardness and grain size is also the same tendency. Wilcox investigated relationship between 
grain size and strength of pure $\mathrm{Ni}$ and several Ni alloys, and showed Hall-Petch relations of each metal ${ }^{31)}$. In case of the pure $\mathrm{Ni}$, relationship between reciprocal of the grain size $d$ $(\mu \mathrm{m})$ and yield strength $\sigma_{y}(\mathrm{MPa})$ can be interpreted as following equation:

$$
\sigma_{y}=14.69+332.50 d^{-0.5}
$$

In this study, the size of minor axis of the fibrous grain of the original Ni matrix was approximately $200 \mathrm{~nm}$ (Fig. 3(b)) and the grain size of the $\mathrm{Ni}-17 \% \mathrm{Cu}$ solid solution formed after welding was approximately $100 \mathrm{~nm}$ (Fig. 7). According to the eq. (1) of pure $\mathrm{Ni}$, yield strengths of pure $\mathrm{Ni}$ with grain size of $200 \mathrm{~nm}$ and $100 \mathrm{~nm}$ is estimated to be $0.8 \mathrm{GPa}$ and $1.1 \mathrm{GPa}$, respectively, and the strength increase due to half reduction of grain size is found to be $138 \%$. The Berkovich hardness value by grain refinement should be 4.32 GPa which is estimated by multiplying $3.13 \mathrm{GPa}$ (hardness of the original $\mathrm{Ni}$ matrix) by 1.38 and this value is lower than that of the obtained $\mathrm{Ni}-17 \% \mathrm{Cu}$ solid solution (6-7 GPa). Wilcox also compared pure $\mathrm{Ni}$ with $\mathrm{Ni}-20 \mathrm{Cr}$ solid solution and showed that slope of Hall-Petch relation of $\mathrm{Ni}-20 \mathrm{Cr}$ solid solution is approximately double larger than that of pure $\mathrm{Ni}^{31)}$. This means that the combined effect of solid-solution strengthening and grain refinement strengthening is possible to induce remarkable hardness increase. No dislocation density measurement was performed in this study. Dor-ram reported that dislocation density of the explosively welded interface was lower than that of region fully away from the interface ${ }^{32}$. Also, as mentioned above section, the interface region is considered to be melted locally. Therefore, work hardening is not a proper reason for hardness increase at the welding interface. Consequently, the remarkable hardness increase detected in $\mathrm{Ni}$-side close to the welding interface is considered to be due to the combined effect of solid-solution strengthening and grain refinement strengthening.

In contrast to Ni-side, constant hardness was detected in $\mathrm{Cu}$-side although $100 \mathrm{~nm}$-diameter-fine grains of $\mathrm{Cu}-27 \% \mathrm{Ni}$ solid solution were formed. Both solid-solution strengthening and grain refinement strengthening should be the reason for hardness increase of $\mathrm{Cu}^{30,33)}$ whereas no hardness increase was detected in $\mathrm{Cu}$-side of the welding interface. The reason for no detection of hardness change in $\mathrm{Cu}$-side is considered to be due to the hardness measurement method. The width of the fine $\mathrm{Cu}-27 \% \mathrm{Ni}$ solid-solution grain region was approximately $500 \mathrm{~nm}$ from the welding interface and this width was smaller than the indentation size (approximately $1 \mu \mathrm{m}$ ). Actually, the distance from the welding interface to most nearest indentation in $\mathrm{Cu}$-side was $550 \mathrm{~nm}$. Hardness increase due to solid-solution strengthening and/ or grain refinement strengthening occurred probably in $\mathrm{Cu}$ side close to the welding interface, however, hardness evaluation could not be performed in this study. The hardness measurement of the extremely narrow region in $\mathrm{Cu}$-side is future work.

Inverse Hall-Petch relation has been observed in several nanocrystalline materials ${ }^{34)}$. This reported that hardness of the materials with nanosized grains decreases contrary to Hall-Petch relation. In this study, grains of $\mathrm{Cu}$ and $\mathrm{Ni}$ in the vicinity of the welding interface were refined to several hun- dred $\mathrm{nm}$ by the welding and hardness increase was observed at this region. This indicates that the combined effect of solidsolution strengthening and grain refinement strengthening strongly influences to hardness than single effect of grain refinement strengthening.

\section{Conclusions}

Joining of $\mathrm{Cu}$ and $\mathrm{Ni}$ plates was performed by using magnetic pulse welding and the interfacial microstructure was examined. The welding interface exhibited characteristic wavy morphology, which is similar to the explosively welded interface. Extremely fine grains of $\mathrm{Cu}-\mathrm{Ni}$ solid solution were formed at both of $\mathrm{Cu}$ and $\mathrm{Ni}$ regions in the vicinity of the welding interface. The width of the fine grain region was different in $\mathrm{Cu}$ - and $\mathrm{Ni}$-sides and it was larger in $\mathrm{Ni}$ side. Remarkable hardness increase was detected in Ni-side close to the welding interface. The hardness increase is assumed to be due to the combined effect of solid-solution strengthening and grain refinement strengthening.

\section{Acknowledgements}

The authors would like to express their thanks to Emeritus Professor Tomokatsu Aizawa and Emeritus Professor Keigo Okagawa of Tokyo Metropolitan College of Industrial Technology for providing experimental facilities and technical assistance. Mr. Toshihiro Suzuki, TOPCON TECHNOHOUSE CORPORATION for his great help for STEM-EDX analysis.

\section{REFERENCES}

1) T. Aizawa: J. JSTP 41 (2000) 265-267.

2) S. Kumai, M. Watanabe and K. Feng: Mater. Sci. Forum 654-656 (2010) 596-601.

3) D. Jaramillo, V.A. Szecket and O.T. Inal: Mater. Sci. Eng. 91 (1987) 217-222.

4) M. Watanabe and S. Kumai: Mater. Trans. 50 (2009) 2035-2042.

5) T. Aizawa, M. Kashani and K. Okagawa: Weld. J. 86 (2007) 119-124.

6) K. Lee, S. Kumai, T. Arai and T. Aizawa: Mater. Sci. Eng. A 471 (2007) 95-101.

7) S.D. Kore, P.P. Date and S.V. Kulkarni: J. Mater. Process. Technol. 208 (2008) 486-493.

8) H. Yu, Z. Fan and C. Li: J. Mater. Process. Technol. 214 (2014) $141-150$.

9) M. Marya and S. Marya: Sci. Technol. Weld. Join. 9 (2004) 541-547.

10) R.N. Raoelison, D. Racine, Z. Zhang, N. Buiron, D. Marceau and M. Rachik: J. Manuf. Process. 16 (2014) 427-434.

11) A. Ben-Artzy, A. Stern, N. Frage and V. Shribman: Sci. Technol. Weld. Join. 13 (2008) 402-408.

12) A. Stern and M. Aizenshtein: Mater. Sci. Technol. 27 (2011) 1809-1813.

13) S. Kumai, M. Watanabe, M. Araki, K. Okagawa and T. Aizawa: J. LMW 49 (2011) 432-440.

14) S. Kakizaki, M. Watanabe and S. Kumai: Mater. Trans. 52 (2011) 1003-1008.

15) Y. Ishii, T. Onzawa, N. Seki and T. Yanagisawa: J. JWS 40 (1971) 523-534.

16) T.B. Massalski: Binary Alloy Phase Diagrams, (ASM international, Ohio, 1990) pp. 1442-1446.

17) M. Watanabe, S. Kumai, G. Hagimoto, Q. Zhang and K. Nakayama: Mater. Trans. 50 (2009) 1279-1285.

18) M. Watanabe and S. Kumai: Mater. Trans. 50 (2009) 286-292.

19) M. Nishida, A. Chiba, Y. Morizono, M. Matsumoto, T. Murakami and 
A. Inoue: Mater. Trans., JIM 36 (1995) 1338-1343.

20) A.S. Bahrani, T.J. Black and B. Crossland: Proc. R. Soc. Lond. A Math. Phys. Sci. 296 (1967) 123-136.

21) G.R. Cowan and A.H. Holtzman: J. Appl. Phys. 34 (1963) 928-939.

22) S.R. Reid: Int. J. Mech. Sci. 16 (1974) 399-413.

23) Y. Mishima, S. Ochiai and T. Suzuki: Acta Metall. 33 (1985) 1161-1169.

24) W.E. Krull and R.W. Newman: J. Appl. Crystallogr. 3 (1970) $519-521$.

25) H. Nosé: J. Phys. Soc. Jpn. 16 (1961) 342.

26) D.G. Morris and M.A. Morris: Mater. Sci. Eng. A 104 (1988) 201-213.

27) F. Salimyanfard, M.R. Toroghinejad, F. Ashrafizadeh and M. Jafari: Mater. Sci. Eng. A 528 (2011) 5348-5355.
28) J. Nishiwaki, T. Kambe, Y. Kedo, Y. Harada, S. Muraishi and S. Kumai: Mater. Sci. Forum 877 (2017) 655-661.

29) T. Sapanathan, R.N. Reoelison, N. Buiron and M. Rachik: Scr. Mater 128 (2017) 10-13.

30) O. Izumi: Hitetsu-zairyou, (Japan Institute of Metals, Sendai, 1987) p. 80 .

31) B.A. Wilcox and A.H. Clauer: Acta Metall. 20 (1972) 743-757.

32) Y. Dor-Ram, B.Z. Weiss and Y. Komem: Acta Metall. 27 (1979) 1417-1429.

33) N. Hansen: Metall. Trans., A, Phys. Metall. Mater. Sci. 16 (1985) 2167-2190.

34) A.M. El-Sherik, U. Erb, G. Palumbo and K.T. Aust: Scr. Metall. Mater. 27 (1992) 1185-1188. 